\title{
Exercise Rehabilitation in Heart Disease: the real "polypill" for primary and secondary prevention
}

\author{
Riabilitazione fisica nel cardiopatico: la vera "polypill" \\ per la prevenzione primaria e secondaria
}

\author{
Massimo F. Piepoli
}

\begin{abstract}
Exercise Rehabilitation in Heart Disease: the real "polypill" for primary and secondary prevention. M.F. Piepoli.

Our society is currently at war against the ominous enemy of chronic disease. Chronic disease presents a heavy burden to society, in terms of both medical costs and human suffering. It is our perception that: 1) much of the medical community underpractises primary prevention as regards appropriate levels of physical activity for health, and 2) much of the research community undervalues the importance of understanding the physiological, genetic and clinical bases of diseases caused by physical inactivity. For many, exercise is viewed solely as a research or diagnostic
\end{abstract}

\begin{abstract}
tool and not as a true weapon against chronic disease. In reality, however, exercise attacks the roots of chronic disease, i.e. physical inactivity. The first step in a common "battle plan" is to convince the medical community that chronic disease is rooted in physical inactivity. In this review, we focus on the biological evidence to date showing how physical inactivity leads to chronic disease. One purpose of this review is to demonstrate that exercise, such as treadmill testing of humans for cardiac dysfunctions, is more than a diagnostic tool but part of disease management itself.

Keywords: exercise, prevention, conditioning.
\end{abstract}

Monaldi Arch Chest Dis 2005; 64: 88-93.

Corresponding author: Massimo F. Piepoli; Heart Failure Unit, Cardiology Unit, Ospedale Guglielmo da Saliceto Polichirurgico; I-29100 Piacenza, Italy; E-mail:m.piepoli@ausl.pc.it

Our society and indeed the world population as a whole is truly at war against a common enemy. That enemy is modern chronic disease: "modern chronic disease" is defined as a disease that is slow in its progress and long in its continuance. ${ }^{1}$ When an individual crosses the threshold called "clinical horizon" and manifests (and is diagnosed with) a multifactorial chronic disease it is generally years after the original causes of the disease have taken effect. The physiological mechanisms underlying the disease have usually been active long before a particular victim is outwardly affected. Major examples of chronic disease are coronary heart disease (including atherosclerosis, heart failure, hypertension, and stroke), obesity, type 2 diabetes, some cancers, osteoporosis, and sarcopenia (frailty in old age as a result of weak muscles).

It would be difficult to find anyone in our society who is exempt from the devastating effects of one or more chronic diseases. Even if an individual does not suffer directly from chronic disease, they likely suffer indirectly as a result of stress from caregiving to others, death of family members or friends, and/or increased health care costs.

Are we winning the war against modern chronic disease? The answer is a resounding "No!" There has been a dramatic increase in the incidence of chronic diseases in the latter part of the $20^{\text {th }}$ century.
Chronic disease conditions cause great human suffering, affecting 90 million Americans and costing nearly U.S. \$0,66 trillion in health care expenses and related lost of productivity in 1990. ${ }^{2}$ Furthermore, this figure may now be approaching U.S. \$1 trillion and stands to further increase dramatically as the baby boom generation ages.

Moreover, we estimate that the cost to society resulting solely from the triad of coronary heart disease, diabetes, and obesity alone is nearly half a trillion dollars! True, we have won some battles in the past few decades; however, such figures indicate that we are still losing the war against modern chronic disease. It may come as a shock to many that the advances made against modern chronic diseases over the past 30 years have come to a halt. The decline in heart disease and stroke deaths 'appears to be possibly going back up.' Previous statistics showing the decreasing percentage of some coronary heart diseases may have masked the urgency of current and imminent future health care problems.

The importance of physical activity in the evolution of the species

The reaction of health care providers and the biomedical establishment to this epidemic has primarily been to apply modern technologies to sta- 
bilise overt clinical problems (e.g. secondary and tertiary prevention). However, since this approach has been largely unsuccessful in reversing the epidemic, we propose that more emphasis should be placed on novel approaches such as primary prevention, which requires attacking the environmental roots of these conditions. In this respect, a strong association exists between the increase in physical inactivity and the emergence of modern chronic diseases in modern industrialized societies. Approximately 250,000 deaths per year in the United States are premature due to physical inactivity. Epidemiological data have established that physical inactivity increases the incidence of at least 17 unhealthy conditions, almost all of which are chronic diseases or considered risk factors for chronic diseases (table 1).

We support the notion that the human genome evolved within an environment of high physical activity. Accordingly, we retain that exercise physicians do not study 'the effect of physical activity' but in reality study the effect of reintroducing exercise into an unhealthy sedentary population that is genetically programmed to expect physical activity. On the basis of healthy gene function, exercise research should thus be viewed from a nontraditional perspective in that the 'control' group should actually be taken from a physically active population and not from a sedentary population with its predisposition to modern chronic diseases. There is an exciting field of exercise biology research that is elucidating the underlying mechanisms by which physical inactivity may predispose individuals to chronic disease conditions, such as mechanisms contributing to insulin resistance and decreased skeletal muscle lipoprotein lipase activity (figure 1). Some findings
Table 1. - Unhealthy conditions precipitated by physical inactivity

- Hypertriglyceridemia
- Hypercholesterolemia
- Obesity
- Type 2 diabetes
- Hyperglycemia
- Insulin resistance
- Increased thrombosis
- Increased resting blood pressure
- Increased risk of myocardial ischemia
- Increased incidence of lethal ventricular arrhythmias
- Decreased cardiac stroke volume, maximal cardiac output
- Progression of heart failure
- Breast and colon cancer
- Osteoporosis
- Sarcopenia
- Back pain
- Gallstone disease
- Decreased psychological well-being

have been surprising and remarkable in that novel signalling mechanisms have been discovered that vary with the type and level of physical activity/inactivity at multiple levels of gene expression. It has been hypothesised that the human genome evolved to support higher metabolic rates and strength activities of a physically active lifestyle. But while the human genome has remained largely unchanged during the past 10,000 years, the recent occurrence of a less physically active lifestyle does not maintain - it has been speculated - the required metabolic

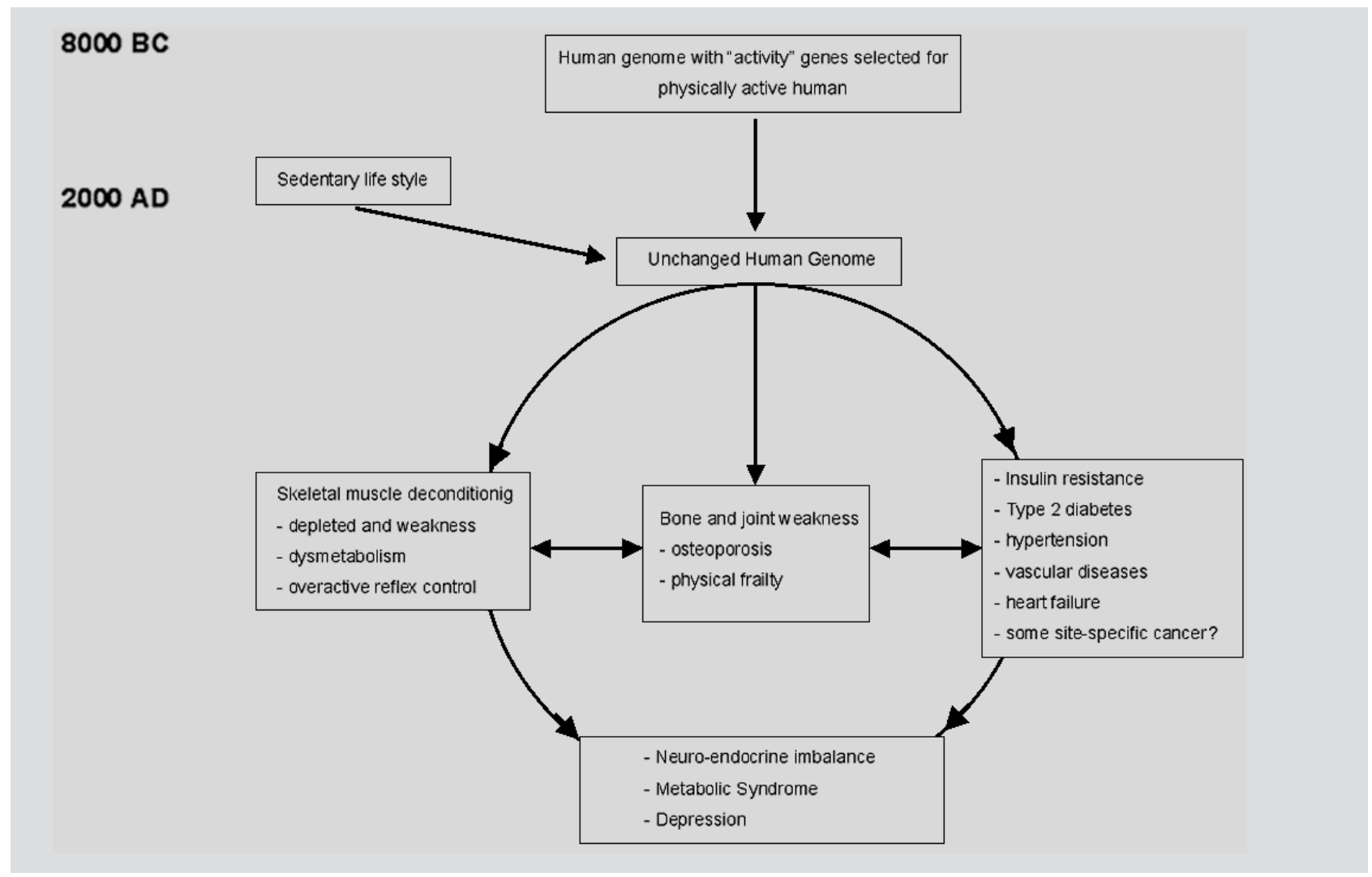

Figure 1. - Biological basis for the hypothesis that the human genome requires physical activity to maintain health. 
fluxes and muscle loading. As a consequence, genes expecting physical activity for normal function have altered their expression such that the resultant phenotype induces a crossing of the threshold of clinical significance whereby overt clinical disorders appear. ${ }^{3}$

Daily physical activity was an integral, obligatory aspect of our ancestor's existence. ${ }^{4}$ Skeletal remains from preagricultural hunter-gatherers showed that they had habitual activity that made them more muscular and stronger than individuals in postagricultural society. 5 Today, most Americans and Europeans are quite weak relative to our ancestors, possibly contributing to the premature onset of physical disability. ${ }^{6}$

Because this area of research is underfunded despite its high impact, the final part of our blueprint for the new millennium calls for the National Health Service to promote and implement major initiatives devoted to the study of the biology of primary prevention of modern chronic diseases.

This would be an investment to avoid bankruptcy of the European health care system and as well would help reduce the extreme human suffering caused by chronic diseases. In short, it would be an investment in the future of health care in the new millennium.

\section{Concept of the polypill}

The growing worldwide burden of cardiovascular disease mandates the development and implementation of effective population-based preventive strategies. ${ }^{7}$ Recently, Wald and $\mathrm{Law}^{8}$ proposed a theoretical cardioprotective "polypill," based on an analysis of the scientific literature (including $>750$ trials with 400,000 participants ${ }^{9}$ ), as a population strategy to combat cardiovascular disease. The investigators boldly claimed that "it would be acceptably safe and, with widespread use, would have a greater impact on the prevention of disease in the Western world than any other single intervention." The formulation was based on an analysis of the 6 components of the pill, which would include: a statin; 3 blood pressure-lowering drugs (e.g. a thiazide, a blocker, and an angiotensin-converting enzyme [ACE] inhibitor), each at half standard dose; folic acid $(0.8 \mathrm{mg})$; and aspirin $(75 \mathrm{mg})$. The strategy was to simultaneously reduce 3 cardiovascular risk factors (low-density lipoprotein [LDL] cholesterol, hypertension, and serum homocysteine), regardless of pretreatment levels combined with the antiplatelet effects of aspirin and the vascular protective effects of an ACE inhibitor and blocker.

The provocative analysis suggested that with a daily dose, this combination therapy would reduce coronary heart disease (CHD) events by $88 \%$ (95\% confidence interval $84 \%$ to $91 \%$ ) and stroke by $80 \%$ (95\% confidence interval $71 \%$ to $87 \%$ ). Omitting any single ingredient other than the statin or blood pressure-lowering drugs had a relatively minor impact on the estimated reduction in CHD and stroke events, respectively. Depending on the precise for- mulation of blood pressure-lowering drugs, the proposed intervention would cause symptoms in $8 \%$ to $15 \%$ of persons taking the pill, warranting withdrawal in 1 to 2 of 100 and causing fatal side effects in $<1$ in 10,000 users. If this calculation is correct, the benefits would substantially outweigh the risk in persons with documented cardiovascular disease and many others at increased risk. However, proponents acknowledged that components of the pill may be unsuitable for persons with some medical conditions (i.e. asthma) or intolerant to aspirin.

To whom should the proposed radical intervention be offered? The investigators recommend it for virtually all patients who have had a previous myocardial infarction or cerebral thrombosis, as well as for patients with angina pectoris, transient ischemic attacks, peripheral arterial disease, and diabetes mellitus. Moreover, because most fatalities from cardiovascular events occur in people aged $\geq 55$ years, the researchers suggest that treating everyone in this age group would prevent nearly all such deaths. They further contend that baseline and serial risk factor profiling is unnecessary, because the prevalence of risk factors is high in Western societies, and aggressive intervention is effective, regardless of the initial levels of risk factors. 10

\section{Pill-popping replaces healthy habits}

But the proposed preventive strategy is purely speculative at this time, and it ignores several major risk factors (i.e. obesity, diabetes, cigarette smoking, physical inactivity) while it relies on a plethora of drugs that essentially substitute for a healthy lifestyle and, as a further objection, the cost would be prohibitive. Furthermore, the problem with this "one-size-fits-all" approach is that some people would be undertreated while others would be overtreated. Administering low-dose aspirin (75 $\mathrm{mg}$ /day), a component of the polypill, to a large population of healthy people would most likely have serious adverse effects, mainly due to hemorrhage. Although evidence to support the use of aspirin in secondary prevention comes from $>100$ randomized trials, the use of aspirin in primary prevention is a physician-guided decision that includes consideration of the patient's age, cardiovascular risk profile, the adverse effects of the drug, and the estimated benefits in reducing the risk of a first myocardial infarction. ${ }^{11}$ Contemporary guidelines for the use of aspirin in primary prevention suggest that the balance of benefits and disadvantages is most favourable in patients at high risk for acute cardiovascular events (i.e. those with a 10 -year risk of $\geq 10 \%)^{12}$

The major limitation of the polypill, however, is that it represents what is a common response of many in the medical community to modern chronic diseases, i.e. the extrapolation of contemporary pharmacotherapies and modern technologies as a first-line strategy to stabilise overt cardiovascular disease. It also gives the wrong message to the population at large-that there is a "quick fix" for cardiovascular health in the form of a magic bullet. Be- 
cause drug therapy and coronary revascularisation have been largely unsuccessful in halting and reversing the epidemic, we argue that more emphasis must be placed on novel approaches to enhance current primary prevention guidelines, which require attacking conventional risk factors and their underlying environmental causes: high-fat and cholesterol diets, cigarette smoking, obesity, and physical inactivity. ${ }^{12,13}$ Recent studies challenge the widely held notion that CHD often ( $\geq 50 \%$ of the time) occurs in the absence of any conventional risk factor, 14 especially when more stringent cut-offs for abnormal blood pressure, cholesterol, and blood glucose levels are employed.

Drug therapy per se also fails to address a major risk factor for cardiovascular disease - physical inactivity and/or low aerobic fitness.15,16 Epidemiologic data have established that physical inactivity increases the incidence of unhealthy conditions, almost all of which are chronic diseases, resulting in approximately 250,000 premature deaths in US each year. ${ }^{13}$ One meta-analysis of 43 studies reported that the relative risk of $\mathrm{CHD}$ in relation to physical inactivity ranged from 1.5 to 2.4 (median value 1.9). ${ }^{17}$ Low aerobic fitness has also been shown to be an independent and more powerful predictor of fatal cardiovascular events than other conventional risk factors. Although recent studies have identified multiple mechanisms by which regular physical activity may decrease morbidity and mortality rates associated with CHD (table 2), perhaps Roberts 18 summarized it best nearly 2 decades ago when he described exercise (running) as "... an agent with lipid-lowering, antihypertensive, positive inotropic, negative chronotropic, vasodilating, diuretic, anorexigenic, weight-reducing, cathartic, hypoglycemic, tranquilizing, hypnotic and antidepressive qualities." In a randomized trial of 101 male patients with stable CHD and an angiographically documented stenosis amenable to percutaneous coronary angioplasty, compared with the latter, a 12-month exercise training program resulted in superior event-free survival and exercise capacity at lower costs. ${ }^{19}$

\section{Beneficial effect of exercise conditioning}

Contemporary guidelines suggest that patients should engage in $\geq 30$ minutes of moderate-intensity physical activity such as brisk walking on most, and preferably all, days of the week. ${ }^{20}$ Randomized trials have now shown that a lifestyle approach to

Table 2. - Potential cardioprotective effects of regular physical exercise

Anti-atherosclerotic

Anti-thrombotic

Anti-ischaemic

Vagomimetic, Sympatholitic

Anti-arrhythmic

- Anti-inflammatory physical activity among previously sedentary persons is feasible and has similar effects on aerobic fitness, body composition, and coronary risk factors compared with a traditional structured exercise program. ${ }^{21}$ Fortunately, a low aerobic fitness level can be improved by regular endurance exercise, augmented lifestyle activity, or both, with each 1-MET increase in exercise capacity conferring an $8 \%$ to $12 \%$ reduction in mortality. 22

\section{Coronary Artery Disease}

The effects of lifestyle change and drug therapy on cardiovascular risk reduction appear to be independent and additive. ${ }^{23}$ Intensive diet and exercise interventions can be highly effective in facilitating coronary risk reduction, complementing and enhancing medications and, in some instances, even outperforming drug therapy. However, the skyrocketing prevalence of obesity, diabetes mellitus, and physical inactivity suggest the need for "real world" interventions that are designed to circumvent and attenuate barriers to sustained lifestyle modification. These efforts go well beyond the physician's office, and require major paradigm shifts that are supported by adequate patient education and encouragement, especially when individually tailored to the patient's readiness to change, 24 to serial monitoring, and to extensive environmental change.

\section{Hypertension}

The impact of lifestyle on the risk of cardiovascular disease has been well established in clinical trials, but these results are often overlooked and underemphasized. In the Dietary Approaches to Stopping Hypertension (DASH) study, ${ }^{25}$ a diet that was rich in fruits and vegetables and low-fat dairy products but low in saturated fat significantly lowered systolic and diastolic pressure to the same extent as observed in trials of drug monotherapy for mild hypertension. The higher the initial blood pressure reading, the greater the effect of the diet on lowering blood pressure. Moreover, it is estimated that a population-wide reduction in systolic or diastolic blood pressure of the magnitude observed with the combination diet would reduce the incidence of CHD and stroke by $15 \%$ and $27 \%$, respectively. Accordingly, the DASH diet may represent an alternative to drug therapy for persons with hypertension, especially when combined with sodium levels of $<100$ $\mathrm{mmol} /$ day. More recently, the PREMIER clinical trial, which incorporated the DASH diet in 1 of 3 intervention groups, demonstrated the feasibility of comprehensive lifestyle modification in achieving substantial decreases in blood pressure, body weight, and sodium intake, and increases in physical fitness in patients with above-optimal blood pressure, including stage 1 hypertension. 26

\section{Diabetes mellitus}

Considerable data also strongly support the role of lifestyle intervention in improving glucose and insulin homeostasis. The effect of changes in lifestyle on the development of type 2 diabetes in 
high-risk overweight subjects with impaired glucose tolerance has been well established.27 A combination of a reduction in body weight (by $\geq 5 \%$ ), in fat intake (to $<30 \%$ of energy intake) and in saturated fat intake (to $<10 \%$ of energy intake) with an increase in fiber intake (to at least $15 \mathrm{~g} / 1,000 \mathrm{kcal}$ ) and in exercise (to $\geq 30$ minutes/day) resulted in a $58 \%$ reduction in the development of diabetes mellitus compared with the usual-care control group, although the average weight loss in response to the intervention was only modest $(3.5 \pm 5.5 \mathrm{~kg}$ by the end of year 2). Similarly, the Diabetes Prevention Program Research Group 28 demonstrated that a lifestyle modification program with the goals of $\geq 7 \%$ weight loss and $\geq 150$ minutes of physical activity per week in overweight patients with impaired fasting glucose resulted in a $58 \%$ reduction in development of diabetes compared with placebo, whereas drug therapy with metformin reduced the risk by only $31 \%$.

\section{Congestive heart Failure}

For many years patients with heart failure were routinely advised to avoid all exertion. Starting from the late 1980s exercise training reports for CHF patients began to surface. These benefits have now been confirmed for many grades and stages of heart failure, and beneficial effects have been shown on symptoms, quality of life, exercise tolerance and many surrogate measures of heart failure severity and complications. In the first randomised controlled trial of exercise training in $\mathrm{CHF}^{2} 9$ it was shown that training could increase exercise tolerance and improve the symptoms of dyspnoea and fatigue. In further studies improvements were seen in autonomic, neurohormonal vascular, endothelial, muscular and ventilatory function. Even the heart was improved. A big step forward was made in 1999 when Belardinelli and colleagues 30 showed that in addition to improvements in quality of life and exercise tolerance mortality was lower after training. Moreover, fewer hospital readmissions for heart failure were seen in the training group. Although it demonstrated a significant reduction in mortality and morbidity, the study was not a prospective trial powered and designed to evaluate this effect, and thus the results can not be considered definitive. It was followed, however, by an individual patient data meta-analysis that has confirmed a significant reduction in both the risk of death and number of hospitalisations for heart failure. We coordinated a collaborative meta-analysis with inclusion criteria covering all randomised parallel group controlled trials of exercise training for at least 8 weeks with individual patient data on survival for at least 3 months. ${ }^{31}$ Nine datasets, totalling 801 patients were identified and analysed; 395 patients received exercise training and 406 were controls. During a mean (S.D.) follow up of 705 (729) days there were 88 $(22 \%)$ deaths in the exercise arm and $105(26 \%)$ in the control arm. Exercise training significantly reduced mortality (hazard ratio $0.65,95 \% \mathrm{p}=0.015$ ). The secondary end point of death or admission to hospital was also reduced $(0.72 ; \mathrm{p}=0.011)$. No statistically significant subgroup specific treatment ef- fect was observed. We can summarise that training in selected CHF patients is beneficial and safe and can reduce mortality and morbidity. It should therefore be recommended for all stable class I-III CHF patients.

\section{Conclusion}

The presumed expense of the polypill, when applied to all subjects aged $>55$ years, would be staggering. If just a fraction of these funds were, instead, used to greatly enhance effective nation-wide primary prevention efforts (e.g. legislation to stop the proliferation of tobacco smoking, increased physical activity in schools and in the community, and promotion of production and consumption of healthy foods), targeting patients of all ages, the results could have a profound effect on risk reduction for generations to come. Nonetheless, entrepreneurial interests, consumer demand, and the incremental benefits of combination drug therapies (i.e. aspirin, statins, blockers, and ACE inhibitors) may fuel the development of a cardioprotective polypill, which may come to fruition in the next few years. We support the recommendation that treatment with the pill, or treatment with its respective components, individually or in combination, be accompanied by the following "User Directions": "Take medication each day in the prescribed dosage, followed or preceded by $\geq 30$ minutes of moderate physical activity, in combination with a low-fat, low-cholesterol diet, weight management, and the avoidance or cessation of cigarette smoking."

\section{References}

1. Dorland's Illustrated Medical Dictionary (25th ed.). Philadel-phia, PA: Saunders, 1974.

2. Hoffman C, Rice D, Sung H-Y. Persons with chronic conditions. JAMA 1996; 276: 1473-1479.

3. Booth FW, Chakravarthy MV, Gordon SE, Spangenburg EE. Waging war on physical inactivity: using modern molecular ammunition against an ancient enemy. $J$ Appl Physiol 2001; 93: 3-30.

4. Cordain L, Gotshall RW, Eaton SB, and Eaton SB III. Physical activity, energy expenditure and fitness: an evolutionary perspective. Int J Sports Med 1998; 19: 328335.

5. Eaton SB, Konner M, and Shostak M. Stone agers in the fast lane: chronic degenerative diseases in evolutionary perspective. Am J Med 1988; 84: 739-749.

6. Spirduso WW, Cronin DL. Exercise dose-response effects on quality of life and independent living in older adults. Med Sci Sports Exerc 2001; 33: S598-S610.

7. Bonow RO, Smaha LA, Smith SC Jr, Mensah GA, Lenfant C. The international burden of cardiovascular disease: responding to the emerging global epidemic. Circulation 2002; 106: 1602-1605.

8. Wald NJ, Law MR. A strategy to reduce cardiovascular disease by more than $80 \%$. BMJ 2003; 326: 1419-1423.

9. Rodgers A. A cure for cardiovascular disease? BMJ 2003; 326: $1407-1408$.

10. Law MR, Wald NJ. Risk factor thresholds: their existence under scrutiny. BMJ 2002; 324: 1570-1576.

11. Eidelman RS, Hebert PR, Weisman SM, Hennekens CH. An update on aspirin in the primary prevention of cardiovascular disease. Arch Intern Med 2003; 163: 2006-2010.

12. Pearson TA, Blair SN, Daniels SR, Eckel RH, Fair JM, 
Fortmann SP, Franklin BA, Goldstein LB, Greenland P, Grundy SM, et al. AHA guidelines for primary prevention of cardiovascular disease and stroke: 2002 update. Consensus panel guide to comprehensive risk reduction for adult patients without coronary or other atherosclerotic vascular diseases. Circulation 2002; 106: 388-391.

13. Booth FW, Gordon SE, Carlson CJ, Hamilton MT, Waging war on modern chronic diseases: primary prevention through exercise biology. J Appl Physiol 2000; 88: 774-787.

14. Hennekens $\mathrm{CH}$, Increasing burden of cardiovascular disease: current knowledge and future directions for research on risk factors. Circulation 1998; 97: 1095-1102.

15. NCEP Expert Panel, Executive Summary of The Third Report of The National Cholesterol Education Program (NCEP) Expert Panel on Detection, Evaluation, And Treatment of High Blood Cholesterol in Adults (Adult Treatment Panel III). JAMA 2001; 285: 2486-2497.

16. Williams PT. Physical fitness and activity as separate heart disease risk factors: a meta-analysis. Med Sci Sports Exerc 2001; 33: 754-761.

17. Powell KE, Thompson PD, Caspersen CJ, Kendrick JS. Physical activity and the incidence of coronary heart disease. Annu Rev Public Health 1987; 8: 253-287.

18. Roberts WC. An agent with lipid-lowering, antihypertensive, positive inotropic, negative chronotropic, vasodilating, diuretic, anorexigenic, weight-reducing, cathartic, hypoglycemic, tranquilizing, hypnotic and antidepressive qualities. Am J Cardiol 1984; 53: 261-262.

19. Hambrecht R, Walther C, Möbius-Winkler S, Gielen S, Linke A, Conradi K, Erbs S, Kluge R, Kendziorra K, Sabri O, Sick P, Schuler G. Percutaneous coronary angioplasty compared with exercise training in patients with stable coronary artery disease. A randomized trial. Circulation 2004; 109: 1371-1378.

20. Thompson PD, Buchner D, Piña IL, Balady GJ, Williams MA, Marcus BH, Berra K, Blair SN, Costa F, Franklin B, et al. Exercise and physical activity in the prevention and treatment of atherosclerotic cardiovascular disease. Circulation 2003; 107: 3109-3116.

21. Dunn AL, Marcus BH, Kampert JB, Garcia ME, Kohl HW III, Blair SN. Comparison of lifestyle and structured interventions to increase physical activity and cardiorespiratory fitness: a randomized trial. JAMA 1999; 281: 327-334.
22. Franklin BA, de Jong A, Kahn JK, McCullough PA. Fitness and mortality in the primary and secondary prevention of coronary artery disease: does the effort justify the outcome? Am J Med Sports 2004; 6: 23-27.

23. Barnard RJ, Di Lauro SC, Inkeles SB. Effects of intensive diet and exercise intervention in patients taking cholesterol-lowering drugs. Am J Cardiol 1997; 79: 1112-1114.

24. Prochaska J, Di Clemente C. Transtheoretical therapy, toward a more integrative model of change. Psych Theory Res Prac 1982; 19: 276-288.

25. Appel LJ, Moore TJ, Obarzanek E, Vollmer WM, Svetkey LP, Sacks FM, Bray GA, Vogt TM, Cutler JA, Windhauser MM, Lin PH, Karanja N. A clinical trial of the effects of dietary patterns on blood pressure. DASH Collaborative Research Group. N Engl J Med 1997; 336: 1117-1124.

26. Writing Group of the PREMIER Collaborative Research Group. Effects of comprehensive lifestyle modification on blood pressure control. JAMA 2003; 289: 2083-2093.

27. Tuomilehto J, Lindström J, Eriksson JG, Valle TT, Hämäläinen H, Ilanne - Parikka P, Keinäen-Kiukaanniemi S, Laaksso M, Louheranta A, Rastas M, Salminen V, Uusitupa M, for the Finnish Diabetes Prevention Study Group. Prevention of type 2 diabetes mellitus by changes in lifestyle among subjects with impaired glucose tolerance. N Engl J Med 2001; 344: 1343-1350.

28. Knowler WC, Barrett-Connor E, Fowler SE, Hamman RF, Lachin JM, Walker EA, Nathan DM, for the Diabetes Prevention Program Research Group. Reduction in the incidence of type 2 diabetes with lifestyle intervention or metformin. N Engl J Med 2002; 346: 393-403.

29. Coats AJ, Adamopoulos S, Meyer TE, Conway J, Sleight P. Effects of physical training in chronic heart failure. Lancet 1990; 335 (8681): 63- 6.

30. Belardinelli R, Georgiou D, Ginzton L, Cianci G, Purcaro A. Effects of moderate exercise training on thallium uptake and contractile response to low-dose dobutamine of dysfunctional myocardium in patients with ischemic cardiomyopathy. Circulation 1998; 97 (6): 553-61.

31. Piepoli MF, Davos C, Francis DP, Coats AJ, ExTraMATCH Collaborative. Exercise training meta-analysis of trials in patients with chronic heart failure (ExTraMATCH). BMJ 2004; 328 (7433): 1.

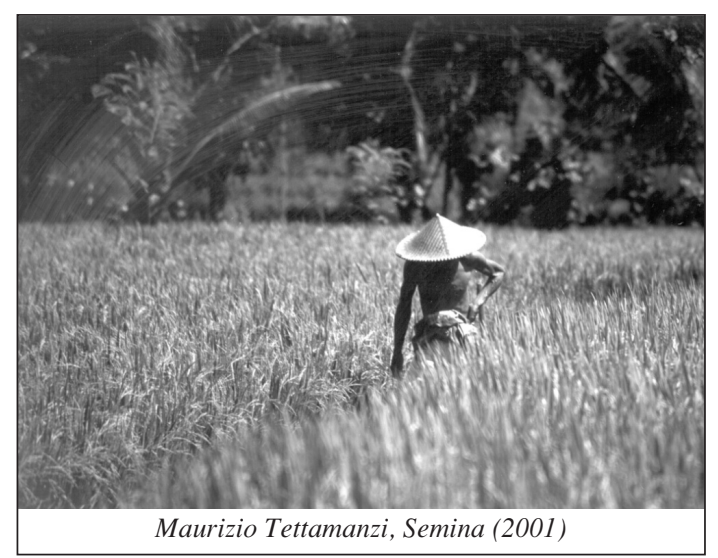

